\title{
Weak topology and a differentiable operator for Lipschitz maps
}

\author{
Abbas Edalat \\ Department of Computing \\ Imperial College London, UK \\ ae@doc.ic.ac.uk
}

\begin{abstract}
We show that the Scott topology induces a topology for real-valued Lipschitz maps on Banach spaces which we call the L-topology. It is the weakest topology with respect to which the L-derivative operator, as a second order functional which maps the space of Lipschitz functions into the function space of non-empty weak ${ }^{*}$ compact and convex valued maps equipped with the Scott topology, is continuous. For finite dimensional Euclidean spaces, where the $L$-derivative and the Clarke gradient coincide, we provide a simple characterisation of the basic open subsets of the $L$ topology in terms of ties or primitive maps of functions. We use this to verify that the L-topology is strictly coarser than the well-known Lipschitz norm topology. We then develop a fundamental theorem of calculus of second order in finite dimensions showing that the continuous integral operator from the continuous Scott domain of non-empty convex and compact valued functions to the continuous Scott domain of ties is inverse to the continuous operator induced by the L-derivative.
\end{abstract}

Key Words: Domain theory, Clarke gradient, Weakest topology, Second order functionals, Hausdorff metric, Fundamental Theorem of Calculus.

\section{The case for Lipschitz maps in computation}

Real-valued Lipschitz maps on Euclidean spaces have a number of fundamental properties that make them into a suitable class of functions in a variety of contexts with wide applications in pure and applied mathematics. For these, they are the appropriate choice of functions in many different areas of computation.

Closed under composition and sitting between the class of continuous functions and those of continuously differentiable functions, Lipschitz maps contain the important class of piecewise polynomial functions, which are widely used in geometric modelling, approximation and interpolation and are supported in MatLab [8]. Lipschitz maps are uniformly continuous and have much better invariant properties than differentiable maps as they are closed under the fundamental min and max operations, and closed under convergence with respect to the sup norm. In the theory and application of ordinary differential equations, Lipschitz maps represent the most fundamental class of maps in view of their basic and essentially unrivalled property that a Lipschitz vector field in $\mathbb{R}^{n}$ has a unique solution in the initial value problem [7].

In a more theoretical direction, Lipschitz maps are, by Rademacher's theorem, differentiable almost everywhere on finite dimensional Euclidean spaces [6, page 148], and by Kirszbraun theorem [24, page 202], enjoy the desirable property that they can be extended from any subset of a Hilbert space to the whole space with the same Lipschitz constant. Lipschitz maps are at the very foundation of nonlinear functional analysis [2] and have been the subject of a hugely growing research in the theory of manifolds including Riemannian surfaces at the forefront of development of mathematics in relation to theoretical physics [3].

In recent years a new notion of derivative for Lipschitz maps has emerged that extends the classical (Fréchet) derivative for continuously differentiable functions and is moreover always defined and continuous with respect to what is in fact the Scott topology on a domain. The Scott topology $[1,25]$, which has proved to be an essential tool in the theory of computation, has now found a new area of application in mathematical analysis.

In 1980's, motivated by applications in non-smooth analysis, optimisation and control theory, Frank Clarke developed a set-valued derivative for real-valued Lipschitz maps on Euclidean spaces, which is now called the Clarke gradient [5]. On finite dimensional Euclidean spaces, the Clarke gradient has non-empty compact and convex subsets of the Euclidean space as its values. For example, the absolute value function, which is not classically differentiable at zero, is a Lipschitz map that has Clarke gradient $[-1,1]$ at zero.

It is of great interest to computer science that the Clarke gradient of a Lipschitz map is upper semi-continuous as 
a function, i.e., it is continuous with respect to the upper topology on the hyperspace of the non-empty compact and convex subsets of the Euclidean space. In finite dimensions, the upper topology coincides with the Scott topology on the hyperspace when it is ordered by reverse inclusion (i.e., its specialisation order). It is however unknown if on infinite dimensional Banach spaces the Clarke gradient, which takes non-empty weak* compact and convex subsets of the dual of the Banach space as its values, remains Scott continuous.

Despite the central place Lipschitz maps occupy in many branches of computation as well as in pure and applied mathematics, they have not been a subject of study in computable analysis to the extent that no mention of Lipschitz maps can be found in the standard texts in computable analysis [30, 34].

In [16], a domain-theoretic derivative was introduced for real-valued functions of the real line, which was later extended to higher dimensions [17, 12] and shown to be mathematically equivalent to the Clarke gradient in finite dimensional spaces [12]. The L-derivative, as the domaintheoretic derivative is now called, has a number of distinct features compared with the Clarke gradient:

(i) The L-derivative $\mathcal{L} f$ of a Lipschitz map $f$ is constructed using finitary differential properties of $f$ that allow a natural way of approximating the L-derivative using domain theory.

(ii) The domain-theoretic setting provides a fundamental theorem of calculus for Lipschitz maps, a duality between primitive maps and their L-derivatives, that extends the classical theorem in calculus for continuously differentiable functions to Lipschitz maps.

(iii) The L-derivative gives rise to a continuous Scott domain with an effective structure for real-valued Lipschitz maps on finite dimensional Euclidean spaces.

(iv) For Lipschitz maps on infinite dimensional Banach spaces, the L-derivative remains Scott continuous, a result not known to hold for the Clarke derivative.

This work has led to a domain-theoretic framework for solving initial value problems $[15,22,18,20]$ including the use of the "rectangular" L-derivative in the second order Euler method [17], a domain-theoretic framework of the implicit and inverse function theorem for Lipschitz functions [19] and a denotational semantics for hybrid systems [21]. A different notion of generalised derivative, called strong derivative, has been recently introduced in [4], which has been employed in a domain-theoretic context for Lipschitz maps to obtain connections with viscosity solutions of Hamiltonian equations.

Two fundamental and related questions arise:
(1) What is the appropriate topology on the space of Lipschitz maps in computation?

(2) Can we obtain a second order typed Fundamental Theorem of Calculus with a continuous derivative operator and a corresponding continuous integral operator as inverses of each other?

We use two different methods, one classical and one domain-theoretic, to tackle and answer the first question:

(i) We obtain the weakest (i.e., the initial) topology on the space of Lipschitz functions that makes the Lderivative operator, which maps the space of Lipschitz functions into the function space of non-empty compact and convex valued maps equipped with its Scott topology, a continuous functional. This is similar to characterising the $C^{1}$ topology on continuously differentiable real-valued maps as the weakest topology that makes the classical Fréchet derivative operator continuous as a second order functional. It is also in tune with the way some of the fundamental topologies, such as the subspace topology, the weak topology of a normed vector space and the weak* topology on its dual are defined.

(ii) We obtain the topology on the space of Lipschitz maps that makes the insertion of these maps onto the set of maximal elements of the domain for Lipschitz maps a topological embedding. This is in line with constructing computational models for classical spaces in mathematics $[11,27]$ by embedding them into the set of maximal elements of suitable domains.

These two approaches lead to an identical result: the Scott topology, both on the hyperspace in (i) and on the domain of Lipschitz maps in (ii) above, induces a topology for maps, called the D-topology, whose intersection with the $C^{0}$ norm topology provides a new topology, called the L-topology, for Lipschitz maps. We show that for Lipschitz maps on finite dimensional Euclidean spaces, the Ltopology is strictly coarser than the well-known Lipschitz norm topology for real-valued Lipschitz maps, which we now describe. Given any metric space $(X, d)$, the collection $\operatorname{Lip}(X, d)$ of bounded real-valued Lipschitz functions on $X$ is equipped with its Lipschitz norm $\|\cdot\|_{\text {Lip }}$ defined as

$$
\|f\|_{\text {Lip }}=\|f\|+\|f\|_{d}
$$

where $\|f\|=\sup \{|f(x)|: x \in X\}$ is the sup norm and $\|f\|_{d}=\sup \{|f(x)-f(y)| / d(x, y): x, y \in X, x \neq y\}$. If $(X, d)$ is complete then so is the Lipschitz norm [32].

The relationship between these topologies is depicted in the diagram below: 


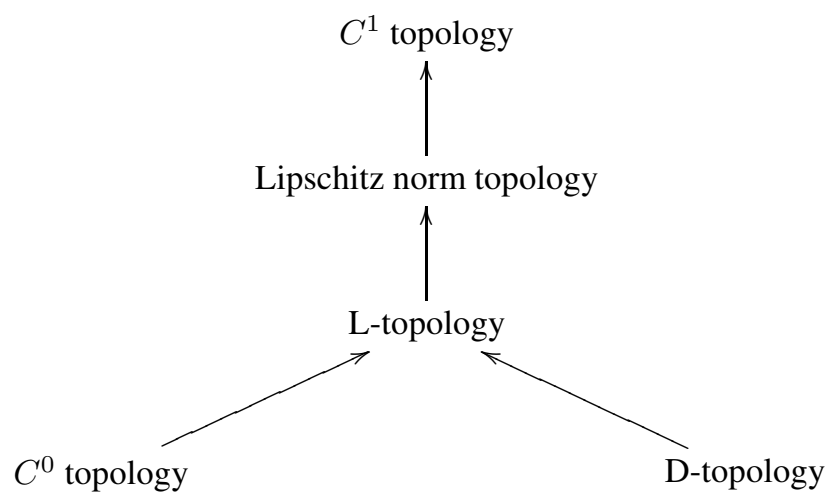

In the finite dimensional case, the set of Lipschitz maps equipped with the L-topology is a Polish space and we derive an elementary characterisation of the basic open subsets of the L-topology in terms of ties or primitive maps. In the one dimensional case, we are able to prove a density lemma for Lipschitz maps which we use to show that the basic open subsets of the L-topology are regular.

Finally, we prove that the domain-theoretic structure of the space of non-empty compact and convex valued maps is preserved after identifying maps that are almost every where the same. This enables us to derive, for the first time using domain theory, a second order typed fundamental theorem of calculus showing that the integral operation and the induced L-derivative operation are continuous inverses of each other.

Due to space limitation, a few proofs including that of Density Lemma 6.2, are skipped here and are given in the full version of the paper [13].

\section{Domain of ties of functions}

In this section we establish our notation, review the elements of the domain-theoretic differential calculus that we need here and extend a number of key results previously only known in dimension one to higher dimensions that will be required in this paper.

We consider continuous maps of type $f: U \rightarrow \mathbb{R}$ where $U \subset \mathbb{R}^{n}$ is an open subset. The set of all such functions is denoted by $(U \rightarrow \mathbb{R})$. The choice of $U$ as an open subset makes the extension of our results to infinite dimensional Banach spaces smooth and uniform. But for finite dimensional spaces, we can also choose $U$ to be a regular compact subset such as $[0,1]^{n}$.

By a domain we mean a directed complete partial order (dcpo). We assume the reader is familiar with the elements of domain theory, in particular the way-below relation, continuous Scott domains, as well as the Scott and Lawson topologies $[1,33,25]$. In any continuous domain $D$ with a basis $B \subset D$, subsets of the form $\uparrow x$, where $x \in B$ form a collection of basic Scott open sets.

Let $\left(\mathbf{C}\left(\mathbb{R}^{n}\right)\right.$, $)$ denote the domain of all non-empty convex and compact subsets of $\mathbb{R}^{n}$, augmented with a least element denoted by $\perp$, ordered by reverse inclusion. The maximal elements are singleton sets $\{x\}$ for $x \in \mathbb{R}^{n}$; for convenience we write any maximal element $\{x\}$ simply as $x$. The dcpo $\left(\mathbf{C}\left(\mathbb{R}^{n}\right), \sqsubseteq\right)$ is a continuous Scott domain as it is a continuous retract [1] of the upper space $\mathbf{U}\left(\mathbb{R}^{n}\right)$, the set of non-empty compact subsets of $\mathbb{R}^{n}$ ordered by reverse inclusion, which is a continuous Scott domain [10]. In fact, the convex hull map $H: \mathbf{U}\left(\mathbb{R}^{n}\right) \rightarrow \mathbf{U}\left(\mathbb{R}^{n}\right)$ that takes any non-empty compact subset to its convex hull is a Scott continuous map with $\mathbf{C}\left(\mathbb{R}^{n}\right)$ as its image. When $n=1$, the dcpo $\mathbf{C}(\mathbb{R})$ is simply the domain $\mathbf{I} \mathbb{R}$ of the non-empty compact intervals of $\mathbb{R}$ ordered by reverse inclusion.

The left and right end points of any non-empty bounded interval $c \subset \mathbb{R}$ is denoted by $c^{-}$and $c^{+}$respectively. Thus, a non-empty compact interval $c \subset \mathbb{R}$ is written in terms of its ends points as $c=\left[c^{-}, c^{+}\right]$. For any topological space $Y$, a Scott continuous function $f: Y \rightarrow \mathbf{I} \mathbb{R}$ is characterized by a lower and an upper semi-continuous functions, $f^{-}, f^{+}$: $Y \rightarrow \mathbb{R}$ respectively, with $f(x)=\left[f^{-}(x), f^{+}(x)\right]$; we write $f=\left[f^{-}, f^{+}\right]$. The scalar product of vectors in $\mathbb{R}^{n}$, i.e., the map - $--: \mathbb{R}^{n} \times \mathbb{R}^{n} \rightarrow \mathbb{R}$ with $x \cdot y=\sum_{i=1}^{n} x_{n} y_{n}$, is extended to a map - - : $\mathbf{C}\left(\mathbb{R}^{n}\right) \times \mathbb{R}^{n} \rightarrow \mathbf{I} \mathbb{R}$ with $b \cdot r=\{z \cdot r \mid z \in b\}$. The Euclidean norm $\|z\|$ of $z \in \mathbb{R}^{n}$ is given by $\|z\|=\sqrt{z \cdot z}$. For a subset $A$ of a topological space, $\mathrm{Cl}(A), A^{\circ}, A^{c}$ denote the closure, interior and complement of $A$ respectively. If $A$ is a subset of a metric space $(Y, d)$ then for any $t>0$ we denote the $t$-neighbourhood of $A$ by $A_{t}=\{x \in Y: \exists y \in A . d(x, y)<t\}$.

For a topological space $Y$ and a depo $D$ with bottom $\perp$, the single-step function $b \chi_{a}: Y \rightarrow D$, where $a \subset Y$ is an open set and $b \in D$, is defined as $b \chi_{a}(x)=b$ if $x \in a$ and $\perp$ otherwise. The domain, $\operatorname{dom}(f)$, of a Scott continuous function $f: Y \rightarrow D$ is given by $\operatorname{dom}(f)=\{x \in Y: f(x) \neq \perp\}$. Since $U$ with its Euclidean topology is a locally compact Hausdorff space, its lattice of open subsets is continuous. It follows by [25, Proposition II-420(iv)] that the space $\left(U \rightarrow \mathbf{C}\left(\mathbb{R}^{n}\right)\right)$ of all Scott continuous functions ordered pointwise is a continuous Scott domain and any $g \in\left(U \rightarrow \mathbf{C}\left(\mathbb{R}^{n}\right)\right)$ can be expressed as the supremum of single-step functions waybelow it: $g=\sup \left\{b \chi_{a}: b \chi_{a} \ll g\right\}$. Lubs of finite and consistent sets of such single-step functions form a basis for $\left(U \rightarrow \mathbf{C}\left(\mathbb{R}^{n}\right)\right)$. Note that we will use the standard notation for step functions in terms of characteristic functions as in [25].

Any single step function of type $U \rightarrow \mathbf{C}\left(\mathbb{R}^{n}\right)$ defines a family of maps of type $U \rightarrow \mathbb{R}$ as follows [12]. We say $f \in$ $(U \rightarrow \mathbb{R})$ has an interval Lipschitz constant $b \in \mathbf{C}\left(\mathbb{R}^{n}\right)$ in a convex relatively compact open subset $a$ with $a \subset U$ if 
for all $x, y \in a$ we have: $b \cdot(x-y) \sqsubseteq f(x)-f(y)$, i.e., $f(x)-f(y) \in b \cdot(x-y)$. The single tie of $b \chi_{a}$, denoted by $\delta\left(b \chi_{a}\right)$, is the collection of all real-valued continuous functions $f$ on $U$ that have an interval Lipschitz constant $b$ in $a$. Note that in our previous work the notation $\delta(a, b)$ was used instead of $\delta\left(b \chi_{a}\right)$. The new notation stresses the connection between a single-tie and its associated singlestep function more explicitly, which is more convenient for expressing the results of this paper. If $f \in \delta\left(b \chi_{a}\right)$ then $f$ is Lipschitz in $a$ with Lipschitz constant $\sup \{\|z\|: z \in b\}$ and the same is true for the extension of $f$ by continuity to $\mathrm{Cl}(a)$.

A tie is any intersection of single-ties. For any indexing set $I$, the family of single-step functions $\left(b_{i} \chi_{a_{i}}\right)_{i \in I}$ is bounded in $\left(U \rightarrow \mathbf{C}\left(\mathbb{R}^{n}\right)\right)$ if $\bigcap_{i \in I} \delta\left(b_{i} \chi_{a_{i}}\right) \neq$ $\emptyset$ [12, Proposition 3.9]. Moreover, if $\sup _{i \in I} b_{i} \chi_{a_{i}} \sqsubseteq$ $\sup _{i \in J} b_{i} \chi_{a_{i}}$, then $\bigcap_{i \in I} \delta\left(b_{i} \chi_{a_{i}}\right) \supseteq \bigcap_{i \in J} \delta\left(b_{i} \chi_{a_{i}}\right)[12$, Corollary 3.12]. It follows that any non-empty tie $\Delta=$ $\bigcap_{i \in I} \delta\left(b_{i} \chi_{a_{i}}\right)$ is uniquely associated with a Scott continuous function $g=\bigsqcup_{i \in I} b_{i} \chi_{a_{i}}$ and we write $\Delta=\delta(g)$. Therefore, $\delta(g)$ is a family of Lipschitz functions whose local Lipschitz properties are expressible by single-ties provided by the single-step functions below $g$. We note that $\delta(g)$ is always non-empty for $n=1$ but can be the empty set in higher dimensions $n>1$. In [17, Section 6], an example of a step function $g$ for dimension $n=2$ is given with $\delta(g)$ the empty set. A function $g \in\left(U \rightarrow \mathbf{C}\left(\mathbb{R}^{n}\right)\right)$ is called integrable if $\delta(g) \neq \emptyset$.

Let $(\mathbf{T}(U), \supseteq)$ be the partial order of ties of continuous functions of type $U \rightarrow X$ ordered by reverse inclusion. The set of L-primitives of a Scott continuous function is precisely the tie associated with it. The L-primitive map is defined by

$$
\begin{aligned}
\int:\left(U \rightarrow \mathbf{C}\left(\mathbb{R}^{n}\right)\right) & \rightarrow \mathbf{T}(U) \\
f & \mapsto \delta(f) .
\end{aligned}
$$

The set $\int f$ is the collection of the $L$-primitives of $f$ and the map $\int$ is continuous with respect to the Scott topologies on $\left(U \rightarrow \mathbf{C}\left(\mathbb{R}^{n}\right)\right)$ and $\mathbf{T}(U)$. In this paper, it is convenient to use $\delta(g)$, which we need to do very often, whenever $g$ is a step function, i.e. the lub of a finite bounded set of singlestep functions, and use $\int g$ for a general Scott continuous function.

The interval Lipschitz constants for a map provide us with its local differential properties, which can be collected to define its global derivative. The L-derivative of a continuous function $f: U \rightarrow \mathbb{R}$ is accordingly defined as the Scott continuous map

$$
\mathcal{L} f: U \rightarrow \mathbf{C}\left(\mathbb{R}^{n}\right),
$$

given by

$$
\mathcal{L} f=\sup \left\{b \chi_{a}: f \in \delta\left(b \chi_{a}\right)\right\}
$$

It is shown in [12] that, in finite dimensional Euclidean spaces, the L-derivative coincides with the Clarke gradient [5]. It is however not known if they do coincide for infinite dimensional Banach spaces; see Section 2.2 below.

Example 2.1 The b-cone. Let $v \in a \subset U, r \in \mathbb{R}$ and let $b$ be any non-empty convex and compact subset of $\mathbb{R}^{n}$. We construct two functions $f^{u}, f^{l}: a \rightarrow \mathbb{R}$ with $f^{u}(v)=f^{l}(v)=r$ and $\mathcal{L} f^{u}(v)=\mathcal{L} f^{l}(v)=b$. The graphs of $f^{u}$ and $f^{l}$ are respectively the upper and lower parts of a cone in $\mathbb{R}^{n+1}$, called the $b$-cone with vertex at $(v, r)$, denoted by $K_{b}(v, r)$. Let $P_{u}^{n}$ be the hyperplane in $\mathbb{R}^{n+1}$ that is perpendicular to $\mathbb{R}^{n}$, passes through $(v, r)$ and contains the unit vector $u \in \mathbb{R}^{n}$. Then $P_{u}^{n}$ intersects $K_{b}(v, r)$ in the hyper-line that stands at angle $\arctan \left((b \cdot u)^{+}\right)$with the $\mathbb{R}^{n}$ hyperplane. For example, when $n=1, b=[-1,1]$ and $x=r=0$, then the $b$-cone is given by the two lines with slopes -1 and 1 through the origin corresponding to the two functions $f^{u}=\lambda x .|x|$ and $f^{l}=\lambda x .-|x|$.

The first order typed Fundamental Theorem of Calculus (FTC) between the L-derivative and primitives gives us the relation [12]:

$$
f \in \int g \Longleftrightarrow g \sqsubseteq \mathcal{L} f .
$$

It is an extension of the classical version of the FTC. In fact, for a continuous real-valued function $g$, we have $f \in \int g$ iff $f$ is $C^{1}$ with $f^{\prime}=g$ where $f^{\prime}$ is the classical (Fréchet) derivative of $f$.

The following notions and results generalise those for dimension one in [16]. We define the function

$$
r:\left(U \rightarrow \mathbf{C}\left(\mathbb{R}^{n}\right)\right) \rightarrow\left(U^{2} \rightarrow \mathbf{I} \mathbb{R}\right)
$$

with the lower and upper parts of $r(g): U^{2} \rightarrow \mathbf{I} \mathbb{R}$ for $g \in\left(U \rightarrow \mathbf{C}\left(\mathbb{R}^{n}\right)\right)$ given by

$(r(g))^{ \pm}:(x, y) \mapsto \begin{cases}\int_{[x, y]}(g \cdot v)^{ \pm} d \mu & {[x, y] \subset \operatorname{dom}(g)} \\ \perp & \text { otherwise }\end{cases}$

where $v=\frac{y-x}{\|y-x\|}$ for $x \neq y$ and $\mu$ is the one dimensional Lebesgue measure on the line segment

$$
[x, y]=\{t x+(1-t) y: 0 \leq t \leq 1\} .
$$

Note that, by the monotone convergence theorem, the map $r$ is Scott continuous.

Lemma 2.2 Suppose $h: U \rightarrow \mathbb{R}$ and $h^{\prime}(x)$ exists for some $x \in U$, then $h^{\prime}(x) \in \mathcal{L}(h)(x)$.

Proof We have $\mathcal{L}(h)(x)=\bigsqcup\left\{b: f \in \delta\left(b \chi_{a}\right) \& x \in a\right\}$. But $h \in \delta\left(b \chi_{a}\right)$ and $x \in a$ imply $h^{\prime}(x) \in b$, and the result follows. 
Lemma 2.3 Let a be a convex open subset of $U$ and $b \in$ $\mathbf{C}\left(\mathbb{R}^{n}\right)$. If for almost all $x \in a$ we have $h^{\prime}(x) \in b$, then $\mathcal{L}(h) \sqsupseteq b \chi_{a}$.

Proof By Rademacher's theorem [6, page 148], for any path connecting $x$ and $y$ we have:

$$
\int_{y}^{x} h^{\prime}(z) \cdot d z=h(x)-h(y) .
$$

In particular, for the path $z:[0,1] \rightarrow[y, x]$ with $z(t)=$ $y+t(x-y)$ we have:

$h(x)-h(y)=\int_{0}^{1} h^{\prime}(y+t(x-y)) \cdot(x-y) d t \in b \cdot(x-y)$.

Hence, $h \in \delta\left(b \chi_{a}\right)$ and the result follows.

Corollary 2.4 Suppose $g \in\left(U \rightarrow \mathbf{C}\left(\mathbb{R}^{n}\right)\right)$ and $h \in(U \rightarrow$ $\mathbb{R})$. If for almost all $x \in \operatorname{dom}(g)$ we have $h^{\prime}(x) \in g(x)$, then $\mathcal{L} h \sqsupseteq g$.

\section{Proposition 2.5 (i) $h \in \int g$ iff}

$$
\forall x, y \in U .(r(g))^{-}(x, y) \leq h(y)-h(x) \leq(r(g))^{+}(x, y)
$$

(ii) If $g$ is an integrable map, then the two functions $\lambda y \cdot(r(g))^{-}(x, y)$ and $\lambda y \cdot(r(g))^{+}(x, y)$ are respectively the least and greatest functions $h \in \int g$ with $h(x)=0$.

(iii) The following two conditions are equivalent:

(a) $g_{1} \sqsubseteq g_{2}$ a.e., i.e., $g_{1}(x) \sqsubseteq g_{2}(x)$ for almost all $x \in U$ with respect to the $n$-dimensional Lebesgue measure on $U$.

(b) $r\left(g_{1}\right) \sqsubseteq r\left(g_{2}\right)$.

(iv) The equivalent conditions (a) and (b) in (iii) imply:

$$
\text { (c) } \int g_{1} \supseteq \int g_{2} \text {. }
$$

(v) If $g_{2}$ is integrable then (c) in (iv) above implies (a) and (b) in (iii).

Proof (i) Suppose $h \in \int g$. Then $\mathcal{L} h \sqsupseteq g$ and $h^{\prime}(x) \in$ $(\mathcal{L} h)(x)$ for a.e. $x \in \operatorname{dom}(g)$. By Rademacher's theorem, integrating along the line segment $[x, y]$ (defined in Equation 6), we get:

$$
\int_{[x, y]}(v \cdot g)^{-} d \mu \leq h(y)-h(x) \leq \int_{[x, y]}(v \cdot g)^{+} d \mu,
$$

as required. On the other hand, suppose the above two inequalities hold. For $x \neq y$, let $y=x+t v$ with $v=(y-x) /(\|y-x\|)$ and $t=\|y-x\|$. Then

$$
\frac{\int_{[x, y]}(v \cdot g)^{-} d \mu}{t} \leq \frac{h(x+t v)-h(x)}{t} \leq \frac{\int_{[x, y]}(v \cdot g)^{+} d \mu}{t} .
$$

By Rademacher's theorem again, $h$ has Fréchet derivative almost everywhere. Therefore, taking the limit $y \rightarrow x$ we obtain for almost all $x \in U$ :

$$
(v \cdot g)^{-}(x) \leq v \cdot h^{\prime}(x) \leq(v \cdot g)^{+}(x) .
$$

Since $v$ is an arbitrary unit vector, it follows that for almost all $x \in U$ we have: $h^{\prime}(x) \in g(x)$. By corollary 2.4, we get $\mathcal{L} h \sqsupseteq g$ as required.

(ii) This follows directly from (i).

(iii) (a) $\Rightarrow$ (b). This follows from monotonicity of $r$.

(b) $\Rightarrow$ (a). From $r\left(g_{1}\right)(x, y) \sqsubseteq r\left(g_{2}\right)(x, y)$ we obtain:

$$
\begin{aligned}
& \int_{[x, y]}\left(g_{1} \cdot v\right)^{-} d \mu \leq \int_{[x, y]}\left(g_{2} \cdot v\right)^{-} d \mu \\
& \int_{[x, y]}\left(g_{1} \cdot v\right)^{+} d \mu \geq \int_{[x, y]}\left(g_{2} \cdot v\right)^{+} d \mu .
\end{aligned}
$$

Thus, for almost all $z \in[x, y]$ with respect to the onedimensional Lebesgue measure on the line segment $[x, y]$ we have the two inequalities: $\left(g_{1} \cdot v\right)^{-}(z) \leq\left(g_{2} \cdot v\right)^{-}(z)$ and $\left(g_{1} \cdot v\right)^{+}(z) \geq\left(g_{2} \cdot v\right)^{+}(z)$. Fix the unit vector $v$. Then by Frobenius theorem $\left(g_{1} \cdot v\right)^{-}(z) \leq\left(g_{2} \cdot v\right)^{-}(z)$ and $\left(g_{1} \cdot v\right)^{+}(z) \geq\left(g_{2} \cdot v\right)^{+}(z)$ for almost all $z \in U$ with respect to the $n$-dimensional Lebesgue measure. Finally, by using Frobenius theorem with spherical integration we obtain $g_{1}(z) \sqsubseteq g_{2}(z)$ for almost all $z \in U$.

(iv) (a) $\Rightarrow$ (c). From $g_{1} \sqsubseteq g_{2}$ a.e. we obtain $r\left(g_{1}\right) \sqsubseteq r\left(g_{2}\right)$. Thus by (i) we obtain $h \in \int g_{1}$ if $h \in \int g_{2}$.

(v) This follows from (i).

\subsection{Lipschitz Domain and computability}

In [11], a domain-theoretic computational model for a classical space $X$ is defined to be a domain $D$ with a topological embedding of $X$ into a subset of maximal elements of $D$ equipped with its relative Scott topology. For a countably based locally compact Hausdorff space, the upper space (the collection of the non-empty compact subsets of the space ordered by reverse inclusion) was proposed as a proto-type model. In this case, as in the case of the domain of formal balls [14] for a complete separable metric space (Polish space), the computational model is an $\omega$-continuous domain. Lawson later showed that an $\omega$-continuous domain is a computational model for a Polish space iff the relative Scott topology and the relative Lawson topology coincide on its the set of maximal elements. [27, 28].

The Scott continuous domain $D^{n}(U)$ for real-valued Lipschitz maps on an open subset $U \subset \mathbb{R}^{n}$ is the set of pairs $(f, g) \in(U \rightarrow \mathbf{I} \mathbb{R}) \times\left(U \rightarrow \mathbf{C}\left(\mathbb{R}^{n}\right)\right)$ that are consistent, i.e., for which there exists a Lipschitz map $h: U \rightarrow \mathbb{R}$ with $f \sqsubseteq h$ and $g \sqsubseteq \mathcal{L} h$; see [12]. For $n=1$, it was shown in [16] that consistency on the basis consisting of 
step functions given by rational numbers is decidable, implying that $D^{n}(U)$ can be given an effective structure. A similar result was given in [17] for $n>1$ in the case of the "rectangular" L-derivative of a function $h: U \rightarrow \mathbb{R}$, whose values, for a fixed coordinate system, at each point $x_{0} \in U$ is the smallest hyper-rectangle in $\mathbb{R}^{n}$ that contains the nonempty compact and convex set $(\mathcal{L} h)\left(x_{0}\right)$. The proofs for the special case of "rectangular" L-derivative in [17] can be extended to show that consistency is decidable for $n>1$, which gives an effective structure for $D^{n}(U)$ in the finite dimensional case. Equipped with an effective structure, the domain $D^{n}(U)$ provides an enumeration of the computable pairs $(f, \mathcal{L} f) \in D^{n}(U)$. Thus, the notions of a computable map and a computable L-derivative are inseparable and are built within the domain of Lipschitz maps.

\subsection{Infinite dimensional case}

The L-derivative can be extended to real-valued functions on any Banach space $X$ [12]; we will only very briefly sketch the way this is done here. Let $U \subset X$ be any open subset of $X$. We consider the differential properties of continuous maps $f: U \rightarrow \mathbb{R}$ with respect to the norm topology on $X$. The L-derivative of $f$ at any point in $U$ where the function is locally Lipschitz will be a non-empty convex and weak* compact subset of the dual space $X^{*}$. Let $\mathbf{C}\left(X^{*}\right)$ denote the dcpo of such subsets ordered by reverse inclusion. Then the notion of tie of a function, which we have seen in the finite dimensional case $\mathbb{R}^{n}$, can be extended to any Scott continuous function $g: U \rightarrow \mathbf{C}\left(X^{*}\right)$ that is expressible as the lub of step functions. These functions form a subdepo $\left(U \rightarrow_{s} \mathbf{C}\left(X^{*}\right)\right)$ of $\left(U \rightarrow \mathbf{C}\left(X^{*}\right)\right)$ which includes all classical functions that map any point of $U$ to a singleton point, i.e. a maximal point, of $\mathbf{C}\left(X^{*}\right)$. Ties of functions are then used to define the L-derivative of any continuous map $f: U \rightarrow \mathbb{R}$ as

$$
\mathcal{L} f: U \rightarrow \mathbf{C}\left(X^{*}\right)
$$

with its values given as in Equation 3. It is shown in [12] that $\mathcal{L} f$ is Scott continuous. It is not known if the Lderivative in the infinite dimensional case coincides with the Clarke gradient or if the Clarke gradient is Scott continuous in the infinite dimensional case.

Note that since the L-derivative can be extended to infinite dimensional Banach spaces, it can be applied to functionals of higher order type such as $(U \rightarrow \mathbb{R}) \rightarrow \mathbb{R}$. In fact, if $U \subset X$ is an open subset of a finite or infinite dimensional Banach space $X$, then the function space $(U \rightarrow \mathbb{R})$ of continuous functions of type $U \rightarrow \mathbb{R}$ with the operator norm forms an infinite dimensional Banach space and therefore the L-derivative is well-defined and Scott continuous on functionals of type $(U \rightarrow \mathbb{R}) \rightarrow \mathbb{R}$ or, inductively, of higher types.

\section{Weak Topology for Lipschitz maps}

In this section we derive a new topology for Lipschitz maps as the weakest topology that makes the the Lderivative operator continuous.

We note that the $C^{1}$ topology on the space of continuously differentiable functions can be characterised as the weakest topology that makes the classical Fréchet derivative operation continuous. In fact, let $C^{0}(U)$ and $C^{1}(U)$ be, respectively, the Banach spaces of continuous functions and continuously differentiable functions on an open subset $U \subset \mathbb{R}^{n}$. Consider the pairing map

$$
\left(\mathrm{Id}, \frac{d}{d x}\right): C^{1}(U) \rightarrow C^{0}(U) \times\left(U \rightarrow \mathbb{R}^{n}\right)
$$

where Id is the identity function and $\frac{d}{d x}$ is the Fréchet derivative operation, i.e. $\left(\mathrm{Id}, \frac{d}{d x}\right)(f)=\left(f, f^{\prime}\right)$. The $C^{1}$ norm topology on $C^{1}(U)$ is precisely the weakest topology such that the above pairing function is continuous.

The above observations lead us naturally to a concrete scheme how to define the weak topology for Lipschitz maps. Instead of the classical Fréchet derivative, we will use the L-derivative. We therefore define the L-topology on the collection $(U \rightarrow \mathbb{R})$, of real-valued continuous functions on $U$, to be the weakest topology on $(U \rightarrow \mathbb{R})$ such that the pairing map

$$
(\mathrm{Id}, \mathcal{L}):(U \rightarrow \mathbb{R}) \rightarrow(U \rightarrow \mathbb{R}) \times\left(U \rightarrow \mathbf{C}\left(X^{*}\right)\right)
$$

with $f \mapsto(f, \mathcal{L} f)$ is continuous, where the function space $(U \rightarrow \mathbb{R})$ in the range of the pairing function above is equipped with its $C^{0}$ norm topology and $\left(U \rightarrow \mathbf{C}\left(X^{*}\right)\right)$ is equipped with its Scott topology.

Let the $D$-topology on $(U \rightarrow \mathbb{R})$ be the weakest topology such that

$$
\mathcal{L}:(U \rightarrow \mathbb{R}) \rightarrow\left(U \rightarrow \mathbf{C}\left(X^{*}\right)\right)
$$

is continuous. Note that the D-topology, like the $\|\cdot\|_{d}$ norm topology in the definition of the Lipschitz norm topology $\|\cdot\|_{L}$ in Equation 1, is not $T_{0}$ as any two functions differing by a constant always belong to the same D-open sets. The L-topology is the intersection (i.e. join) of the $C^{0}$ topology and the D-topology on $(U \rightarrow \mathbb{R})$ and is Hausdorff.

Since the L-derivative of a $C^{1}$ function coincides with its Fréchet derivative, it follows that the $C^{1}$ topology on $C^{1}(U)$ is precisely the relative L-topology for the subspace $C^{1}(U)$.

The L-topology has also a domain-theoretic characterization as follows. The domain $D(U)$ for real-valued Lipschitz maps is the subdomain of the consistent pairs of elements in $\left(U \rightarrow_{s} \mathbb{R}\right) \times\left(U \rightarrow_{s} \mathbf{C}\left(X^{*}\right)\right)$, where $U \subset X$ is an open subset and $X$ is $\mathbb{R}^{n}$ or an infinite dimensional Banach space. 
Proposition 3.1 The L-topology on $(U \rightarrow \mathbb{R})$ is precisely the topology that makes the insertion map

$$
f \mapsto(f, \mathcal{L} f):(U \rightarrow \mathbb{R}) \rightarrow D(U)
$$

a topological embedding with respect to the Scott topology on $D(U)$, i.e., the L-topology is the topology that makes $D(U)$ a computational model.

Proof We first note that for each $f \in(U \rightarrow \mathbb{R})$, the pair $(f, \mathcal{L} f) \in D(U)$ is maximal [12, Proposition 5.8] and that the insertion map is one to one. Next we note that the function space $(U \rightarrow \mathbb{R})$, equipped with its sup norm, is homeomorphic with the subset of maximal elements of $(U \rightarrow \mathbf{I} \mathbb{R})$, equipped with its relative Scott topology, under the correspondence $f \mapsto \lambda x .\{f(x)\}$; see [16, Theorem 2.2]. The statement that the L-topology is the weakest topology that makes the L-derivative $\mathcal{L}: f \mapsto(f, \mathcal{L} f)$ : $(U \rightarrow \mathbb{R}) \times\left(U \rightarrow \mathbf{C}\left(X^{*}\right)\right)$ continuous is equivalent to the assertion that the insertion map is continuous and that it is an open map.

\section{L-topology in finite dimensions}

When $X=\mathbb{R}^{n}$, the pairing map reduces to:

$$
(\mathrm{Id}, \mathcal{L}):(U \rightarrow \mathbb{R}) \rightarrow D^{n}(U)
$$

where $D^{n}(U) \subset(U \rightarrow \mathbb{R}) \times\left(U \rightarrow \mathbf{C}\left(\mathbb{R}^{n}\right)\right)$ is a continuous Scott domain. Since the space of Lipschitz maps equipped with the L-topology is precisely the set of maximal elements of $D^{n}(U)$, it follows that this space is a Polish space, i.e., a separable completely metrizable topological space [25, chapter V-6].

Furthermore, the L-topology will have an elementary characterisation in terms of ties of functions: the Dtopology has a basis consisting of subsets of the form $\breve{\delta}(g):=\mathcal{L}^{-1}(\uparrow g)$ for any step function $g \in\left(U \rightarrow \mathbb{R}^{n}\right)$ with $\uparrow g \neq \emptyset$.

We note that if $g=\sup _{i \in I} b_{i} \chi_{a_{i}}$, for a finite indexing set $I$, then $\uparrow g=\bigcap_{i \in I} \uparrow\left(b_{i} \chi a_{i}\right)$. Since, by FTC (Equation 4), $\delta\left(b \chi_{a}\right)=\mathcal{L}^{-1}\left(\uparrow\left(b \chi_{a}\right)\right)$, it follows that $\breve{\delta}\left(b \chi_{a}\right) \subset \delta\left(b \chi_{a}\right)$ and more generally for any step function $g \in\left(U \rightarrow \mathbb{R}^{n}\right)$ :

$$
\breve{\delta}(g) \subset \delta(g) .
$$

The countable collection of step functions of the form $\bigcup_{1 \leq i \leq m}\left(b_{i} \chi_{a_{i}}\right)$ where, for $1 \leq i \leq m$, the subset $a_{i}$ is the interior of a convex rational polyhedron whereas the subset $b_{i}$ is the closed hull of a rational convex polyhedron, provides a countable basis of the Scott topology on $\left(U \rightarrow \mathbf{C}\left(\mathbb{R}^{n}\right)\right)$. Since the $C^{0}$ norm topology is second countable, it follows that the D-topology and thus its intersection with the $C^{0}$ norm topology are both second countable.
In this section and in Section 6, closure and interior of subsets are meant to be with respect to the L-topology.

Proposition 4.1 Any tie is closed in the L-topology.

Proof Since a tie is an intersection of single-ties, it is sufficient to show the statement for a single-tie $\delta\left(b \chi_{a}\right)$. Since the L-topology is second countable, it suffices to prove the closure property for sequences. Let $\left(f_{i}\right)_{i>0}$ be a sequence in $\delta\left(b \chi_{a}\right)$ which converges to a function $f: U \rightarrow \mathbb{R}$ in the L-topology and thus in particular in the $C^{0}$ norm topology. Then, for each $i \geq 0$, we have: $b \cdot(x-y) \sqsubseteq f_{i}(x)-f_{i}(y)$. From the compactness of $b \cdot(x-y)$, we conclude by taking the limit that for all $x, y \in a$ we have: $b \cdot(x-y) \sqsubseteq$ $f(x)-f(y)$ as required.

From Equation 7, we conclude:

Corollary 4.2 If $g$ is a step function, then: $\mathrm{Cl}(\breve{g}) \subset \delta(g)$.

Corollary 4.3 The L-topology is the weakest topology on $(U \rightarrow \mathbb{R})$ such that the pairing map

$$
(I d, \mathcal{L}):(U \rightarrow \mathbb{R}) \rightarrow(U \rightarrow \mathbb{R}) \times\left(U \rightarrow \mathbf{C}\left(\mathbb{R}^{n}\right)\right)
$$

is continuous, where the function space $(U \rightarrow \mathbb{R})$ in the range of the pairing function above is equipped with its $C^{0}$ norm topology and $\left(U \rightarrow \mathbf{C}\left(\mathbb{R}^{n}\right)\right)$ is equipped with its Lawson topology.

Proof The Scott topology on $\left(U \rightarrow \mathbf{C}\left(\mathbb{R}^{n}\right)\right)$ is refined by the Lawson topology by taking $\uparrow g$ as sub-basic closed subsets for all step functions $g$. But $\mathcal{L}^{-1}\left(\uparrow b \chi_{a}\right)=\delta\left(b \chi_{a}\right)$ is closed in the L-topology by Corollary 6.4.

We say that the way-below relation in a continuous domain is meet-stable if $x \ll y$ and $x \ll z$ imply $x \ll y \sqcap z$ for all $x, y$ and $z$ in the domain. Recall that a space is called coherent if its lattice of open sets is continuous with a meetstable way-below relation $[1,25]$.

Lemma 4.4 Let $D$ be a continuous Scott domain with a meet-stable way-below relation and $Y$ a coherent topological space. Then for any open set $a \subset Y$ and $s \in D$ we have $\uparrow\left(s \chi_{a}\right)=\bigcup\left\{\uparrow\left(t \chi_{b}\right): a \ll b \& s \ll t\right\}=\bigcup\left\{\uparrow\left(t \chi_{b}\right): a \ll\right.$ $b \& s \ll t\}$.

Proof We show the first equality from which the second follows easily. Since $Y$ is a coherent space and $D$ is a continuous Scott domain, we have the following relation [23]:

$$
s \chi_{a} \ll f \Longleftrightarrow a \ll f^{-1}(\uparrow s)
$$

Thus, $a \ll b \& s \ll t$ implies $\uparrow\left(t \chi_{b}\right) \subset \uparrow\left(s \chi_{a}\right)$. It remains to show the reverse inclusion. Let $\left(s \chi_{a}\right) \ll f$. Then, 
since $D$ is a continuous domain, there exists a step function $\bigsqcup_{i \in I} s_{i} \chi_{a_{i}}$, where $I$ is a finite indexing set, with

$$
s \chi_{a} \ll \bigsqcup_{i \in I} s_{i} \chi_{a_{i}} \ll f .
$$

From $s \chi_{a} \ll \bigsqcup\left\{s_{i} \chi_{a_{i}}: 1 \leq i \leq m\right\}$, by Equation 8, we get $a \ll \bigcup_{J \subset I}\left\{\bigcap_{j \in J} a_{j}: s \ll \bigsqcup_{j \in J} s_{j}\right\}$. Since the way-below relation in $D$ is meet-stable, we have $s \ll$ $\prod_{J \subset I}\left\{\bigsqcup_{j \in J} s_{j}: s \ll \bigsqcup_{j \in J} b_{j}\right\}$. Let $s^{\prime} \in D$ be such that $s \ll s^{\prime} \ll \prod_{J \subset I}\left\{\bigsqcup_{j \in J} s_{j}: s \ll \bigsqcup_{j \in J} b_{j}\right\}$. Also let $a^{\prime}$ be an open subset of $Y$ with

$$
a \ll a^{\prime} \ll \bigcup_{J \subset I}\left\{\bigcap_{j \in J} a_{j}: s \ll \bigsqcup_{j \in J} s_{j}\right\} .
$$

Then we have $s \chi_{a} \ll s^{\prime} \chi_{a^{\prime}} \ll f$, which completes the proof.

Since finite intersection distributes over arbitrary union, we can conclude with the same assumptions on $Y$ and $D$ :

Corollary 4.5 For any step function $g \in(Y \rightarrow D)$ we have:

$$
\begin{gathered}
\uparrow g=\bigcup\{\uparrow h: g \ll h \text { step function }\} \\
=\bigcup\{\uparrow h: g \ll h \text { step function }\} .
\end{gathered}
$$

These results now translate to basic L-open subsets.

Corollary 4.6 We have for any step function $g$ :

(i) $\breve{\delta}(g)=\bigcup\{\delta(h): g \ll h$ step function $\}$.

(ii) $\breve{\delta}(g)=\bigcup\{\breve{\delta}(h): g \ll h$ step function $\}$.

\section{L-topology and Lipschitz norm}

Recall the definition of the Lipschitz norm in Section 1. In finite dimensions we can show the following:

Theorem 5.1 The L-topology is coarser than the Lipschitz norm topology in finite dimensions.

Proof Let $f \in \breve{\delta}\left(b \chi_{a}\right)$ for some single-step function $b \chi_{a} \in$ $\left(U \rightarrow \mathbf{C}\left(\mathbb{R}^{n}\right)\right)$. We will find a neighbourhood of $f$ in the Lipschitz norm topology that is contained in $\breve{\delta}\left(b \chi_{a}\right)$. We have $f \in \delta\left(d \chi_{c}\right)$ for some $a \ll c$ and $b \ll d$. Let $e$ be such that $b \ll e \ll d$. Then there exists $t>0$ such that $d_{t} \subset e$. (Recall that $A_{t}$ is the $t$-neighbourhood of a set $A$.) It follows that for all $x, y \in c$ with $x \neq y$ we have $(d \cdot(x-y))_{t\|x-y\|} \subset e \cdot(x-y)$. We have $f(x)-f(y) \in$ $d \cdot(x-y)$ for all $x, y \in \mathrm{Cl}(c)$. Consider any Lipschitz map $h$ with $\|f-h\|_{L}<t$. Then, $\mid(h(x)-h(y))-(f(x)-$ $f(y))|=|(h(x)-f(x))-(h(y)-f(y)|\leq t| x-y \mid$ for all $x, y \in \mathrm{Cl}(c)$. It follows that $h(x)-h(y) \in e \cdot(x-y)$ and thus $h \in \delta\left(e \chi_{c}\right) \subset \breve{\delta}\left(b \chi_{a}\right)$.
Next, we show that the L-topology is strictly coarser than the Lipschitz norm topology in finite dimensions. To do this, we need to construct a counter-example for the assertion that the L-topology is finer than the Lipschitz norm topology in the neighbourhood of a Lipschitz function.

Our construction is non-trivial. We use a onedimensional map that has a non-trivial interval as its Lderivative, equivalently Clarke's gradient, at all points of an interval. The existence of such a map is proved in [29, Proposition 1.9]. For our counter-example, however, we construct below a one-parameter family of Lipschitz maps $f_{k}:[0,1] \rightarrow \mathbb{R}$ for $k \in[0,2]$ such that $\mathcal{L} f_{k}(x)=[0,1]$ for all $x \in[0,1]$ with the property that, as $k \nearrow 1$, we have $f_{k} \rightarrow f_{1}$ in the L-topology but not in the Lipschitz norm topology.

For $k \in[0,2]$, we let $f_{k}=\lambda x \cdot \int_{0}^{x} \chi_{A_{k}} d \mu$ where $\mu$ is the Lebesgue measure and the measurable set $A_{k}$ satisfies:

(i) for any interval $I \subset[0,1]$ of length $\ell(I)>0$ we have: $0<\mu\left(A_{k} \cap I\right)<\ell(I)$, and,

(ii) $\mu\left(A_{k}\right)=k / 2$.

Property (i) above says that $A_{k}$ splits all non-trivial intervals; see [26] for the existence of such sets in a general setting.

The set $A_{k}$ can be constructed as the countable union of a double family of Cantor sets that are obtained in a sequence of stages. When $k>0$, these Cantor sets will have positive Lebesgue measure.

We first adopt the following uniform scheme, similar to the construction of the standard Cantor set, to construct a Cantor set of Lebesgue measure $s \geq 0$ in a compact interval of length $r>0$ with $r \geq s$. In the first stage the symmetrically placed middle open interval of length $(r-s) / 3$ is removed, then in the remaining left and right closed intervals, the two middle open intervals each of length $(r-s) / 9$, and so on. The total Lebesgue measure of the countable set of removed intervals is thus $\frac{r-s}{3}\left(1+\frac{2}{3}+\frac{4}{9}+\cdots\right)=r-s$. Thus the Cantor set has Lebesgue measure $r-(r-s)=s$.

Now we use our uniform scheme to construct $A_{k}$. In the first stage, a Cantor set of measure $k / 4$ is constructed on $[0,1]$. Therefore, the first middle interval, denoted by $C$, to be removed has length $\left(1-\frac{k}{4}\right) / 3$, the next two middle intervals to be removed, denoted by $L C$ and $R C$, are in the remaining two intervals $L$ and $R$ on the left and right respectively and have each length $\left(1-\frac{k}{4}\right) / 9$, and so on.

Then in each previously removed interval a new Cantor set is constructed so that the total measure of the countable union of the new Cantor sets is $k / 8$. This is done by constructing a Cantor set of measure $k / 16$ in $C$, then constructing two Cantor sets each of measure $k /(4 \times 16)$ (i.e. with total measure $k / 32$ ) in the left and the right middle intervals, namely $L C$ and $R C$, then constructing four Cantor sets each of measure $k /(4 \times 64)$ (i.e, with total measure 
$k / 64)$ in the middle intervals $L L C, L R C, R L C, R R C$ of $L L, L R, R L, R R$ respectively, and so on.

The procedure is then repeated ad infinitum so that a Cantor set is constructed in any previously removed interval. The set $A_{k}$ will be the countable union of the countable unions of Cantor sets constructed at each stage. These Cantor sets are dense in $[0,1]$ : any subinterval of $[0,1]$ contains one of these Cantor sets. We also have $\mu\left(A_{k}\right)=$ $\frac{k}{4}+\frac{k}{8}+\frac{k}{16} \cdots=\frac{k}{2}$, so that $0<\mu\left(A_{k}\right)<1$ for $k \in(0,2)$.

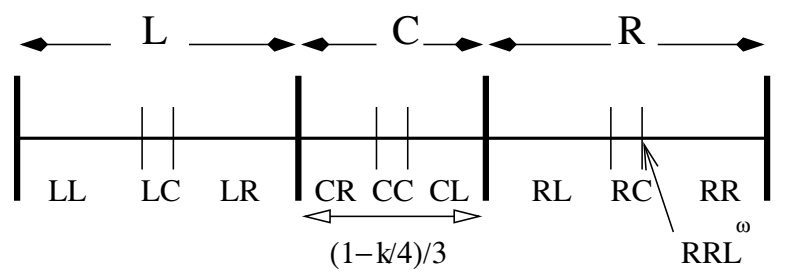

Our labelling scheme for the intervals in the above construction is an instance of a general method in symbolic dynamics [9]. For a given $k \in[0,2]$, we represent each point of $[0,1]$ by an infinite sequence of $L, C, R$, denoting the position of the the point on the Left, Center or Right interval at each stage of construction process, i.e., putting $\Sigma=\{L, C, R\}$, we have a surjection $P_{k}: \Sigma^{\omega} \rightarrow[0,1]$ that takes any sequence to a point in $[0,1]$. As each new interval is a contracting affine image of a previous interval, it follows that for $s \in \Sigma^{\omega}$, we have $P_{k}(s)=A(s)+B(s) k$ where $A, B: \Sigma^{\omega} \rightarrow[0,1]$.

Furthermore, by construction, $f_{k}(x)=\int_{0}^{x} \chi_{A_{k}} d \mu$ is the sum of the Lebesgue measure of the Cantor sets constructed in $[0, x)$. Since the Lebesgue measure of each such Cantor set is a multiple of $k$, we have: $f_{k}\left(P_{k}(s)\right)=D(s) k$ where $D: \Sigma^{\omega} \rightarrow[0,1]$. Note that for all $x \in[0,1]$, we have: $f_{0}(x)=0$ (each Cantor set that is constructed has Lebesgue measure zero) whereas $f_{2}(x)=x$ (the sum of the Lebesgue measures of all constructed Cantor sets in $[0,1]$ is 1 ).

Lemma 5.2 For all $k \in(0,2)$ and $x \in[0,1]$, we have: $\mathcal{L}\left(f_{k}\right)(x)=[0,1]$.

Proof This is an instance of [29, Proposition 1.9].

It follows that for each $k \in[0.2]$, the map $f_{k}$ is Lipschitz with Lipschitz constant 1.

Lemma 5.3 We have $f_{k} \rightarrow f_{k_{0}}$ in the L-topology as $k \nearrow$ $k_{0}$, for any $k_{0} \in(0,2]$.

Proof Since $\mathcal{L} f_{k}=[0,1]$ for any $k \in(0,2)$, it follows that $\mathcal{L} f_{k} \rightarrow \mathcal{L} f_{k_{0}}$ as $k \nearrow k_{0}$. Thus it remains to show that $f_{k} \rightarrow f_{k_{0}}$ in the sup norm as $k \nearrow k_{0}$. We will show that for any fixed $x \in[0,1]$, the function $k \mapsto f_{k}(x)$ is strictly increasing with $k$ and that $f_{k}(x) \rightarrow f_{k_{0}}(x)$ pointwise as $k \nearrow k_{0}$. Since $f_{k}$ is continuous for all $k \in[0,2]$, the result will then follow a well known result in analysis [31, see 7.13]. Since $f_{k}$, being Lipschitz, is continuous for each $k \in[0,2]$, it suffices to show the above two properties for a dense subset of $[0,1]$. To show that $f_{k}(x)<f_{k_{0}}(x)$ for $k<k_{0}$, we consider the dense subset $\left\{x_{t}: t \in \Sigma^{*}\right\}$, where $x_{t}:=P_{k}\left(t R L^{\omega}\right)$. Since for each fixed $k \in[0,1]$, the map $f_{k}$ is increasing and for $k<k_{0}$ by construction we have $P_{k_{0}}\left(t R L^{\omega}\right)<P_{k}\left(t R L^{\omega}\right)$, we obtain:

$$
\begin{gathered}
f_{k}\left(x_{t}\right)=f_{k}\left(P_{k}\left(t R L^{\omega}\right)\right)=D\left(t R L^{\omega}\right) k<D\left(t R L^{\omega}\right) k_{0} \\
=f_{k_{0}}\left(P_{k_{0}}\left(t R L^{\omega}\right)\right)<f_{k_{0}}\left(P_{k}\left(t R L^{\omega}\right)\right)=f_{k_{0}}\left(x_{t}\right),
\end{gathered}
$$

which proves the first assertion. For the second assertion, we consider the dense subset $\left\{y_{t}: t \in \Sigma^{*}\right\}$, where $y_{t}:=P_{k_{0}}\left(t L R^{\omega}\right)$. Since for $k \leq k_{0}$ we have $P_{k}\left(t L R^{\omega}\right) \leq$ $P_{k_{0}}\left(t L R^{\omega}\right)$, we obtain:

$$
D\left(t L R^{\omega}\right) k \leq f_{k}\left(y_{t}\right) \leq f_{k_{0}}\left(y_{t}\right)=D\left(t L R^{\omega}\right) k_{0},
$$

and it follows that $f_{k}\left(y_{t}\right) \rightarrow f_{k_{0}}\left(y_{t}\right)$ as $k \nearrow k_{0}$, which proves the second assertion, completing the proof.

Using the family $\left(f_{k}\right)_{0 \leq k \leq 2}$ we can prove:

Proposition 5.4 There exists no open set of the L-topology around the map $f_{1}$, as constructed above, that is contained in the open ball $\left\{f:\left\|\mid f-f_{1}\right\|_{\text {Lip }}<1\right\}$ of unit radius with respect to the Lipschitz norm around $f_{1}$, i.e., the L-topology is strictly coarser than the Lipschitz norm topology.

\section{L-topology in dimension one}

In dimension one ( $n=1)$, we assume, for convenience, that $U \subset \mathbb{R}$, the domain of our continuous functions in $(U \rightarrow \mathbb{R})$, is a compact interval. We are able to show here that a basic L-open subset $\breve{\delta}(g)$ is the interior (with respect to the L-topology) of the associated tie $\delta(g)$. Recall that in dimension one, any Scott continuous function $g \in(U \rightarrow \mathbf{I} \mathbb{R})$ is integrable, i.e., there exists $h \in \int g$ with $g \sqsubseteq \mathcal{L} h$. In fact, it is shown in [16, section 6] that given any lower semi-continuous function $u: U \rightarrow \mathbb{R}$ there exists a least function $s(u, g): \operatorname{dom}(g) \rightarrow \mathbb{R}$ such that $u \leq s(u, g)$ and $g \sqsubseteq \mathcal{L} s(u, g)$. Furthermore, if $g: U \rightarrow \mathbf{I} \mathbb{R}$ is a step function and $u$ is the lower part of a step function of type $U \rightarrow \mathbf{I} \mathbb{R}$, then $s(u, g)$ is a piecewise linear map in each connected component of $g$; see [15, Section 3]. In the following we deduce that when $u$ is fixed, the least function $s(u, g)$ will depend continuously on $g$ with respect to the metric induced on step functions by the Hausdorff metric.

Recall that our basis elements for the L-topology are given in terms of step functions $g$ with $\uparrow g \neq \emptyset$. This means that if two adjacent intervals in $\operatorname{dom}(g)$, each with a constant value for $g$, are just touching then their corresponding values will have non-empty intersection. Thus, the 
connected components of $\mathrm{Cl}(\operatorname{dom}(g))$ have disjoint open neighbourhoods. Dealing with these connected components separately, let $\left(U \rightarrow^{u} \mathbf{I} \mathbb{R}\right)$ be the collection of step functions $g \in(U \rightarrow \mathbf{I} \mathbb{R})$ with $\uparrow g \neq \emptyset$ such that $\mathrm{Cl}(\operatorname{dom}(g))$ has a single connected component and $\operatorname{dom}(g) \cap \operatorname{dom}(u) \neq \emptyset$. For $g=\left[g^{-}, g^{+}\right] \in\left(U \rightarrow^{u} \mathbf{I} \mathbb{R}\right)$, let

$$
\begin{gathered}
S(u, g): U \times U \rightarrow \mathbb{R} \\
(x, y) \mapsto \begin{cases}u(y)+\int_{y}^{x} g^{-}(t) d t & x \geq y \\
u(y)-\int_{x}^{y} g^{+}(t) d t & x<y\end{cases}
\end{gathered}
$$

Let $P_{g}$ be the partition of the interval $\operatorname{dom}(g) \cup \operatorname{dom}(u)$ obtained as the common refinement of the partition induced by the step function $g$ and that by the piecewise constant map $u$ such that in each interval in $P$ the values of $g$ and $u$ are constant (note that for $g$ these values are non-empty compact intervals and for $u$ they are real numbers). Then, as in [15], we have:

$$
\begin{gathered}
s(u, g)=\lambda x . \\
\max \{u(x)\} \cup\left\{\limsup _{y \rightarrow z} S(u, g)(x, y): z \in P_{g} \cap \operatorname{dom}(u)\right\} .
\end{gathered}
$$

Consider $\left(U \rightarrow^{u} \quad \mathbf{I} \mathbb{R}\right)$ with the partial distance function induced from the Hausdorff metric on $\mathbf{I} \mathbb{R}$, namely: $d\left(g_{1}, g_{2}\right)=\sup \left\{d_{H}\left(g_{1}(x), g_{2}(x)\right): x \in \operatorname{dom}\left(g_{1}\right) \cap\right.$ $\left.\operatorname{dom}\left(g_{2}\right)\right\}$ and consider the partial maps in $U^{2} \rightarrow \mathbb{R}$ with their partial sup norm: $\left\|f_{1}-f_{2}\right\|=\sup \left\{\left|f_{1}(x)-f_{2}(x)\right|\right.$ : $\left.x \in \operatorname{dom}\left(f_{1}\right) \cap \operatorname{dom}\left(f_{2}\right)\right\}$. We then have:

\section{Lemma 6.1 The functions}

(i) $g \mapsto S_{(u, g)}:\left(U \rightarrow^{u} \mathbf{I} \mathbb{R}\right) \rightarrow\left(U^{2} \rightarrow \mathbb{R}\right)$

(ii) $g \mapsto s_{(u, g)}:\left(U \rightarrow^{u} \mathbf{I} \mathbb{R}\right) \rightarrow(U \rightarrow \mathbb{R})$

are continuous with respect to partial distance on $\left(U \rightarrow^{u}\right.$ $\mathbf{I R )}$ and the partial sup norm on $\left(U^{2} \rightarrow \mathbb{R}\right)$.

Proof Note that is $g^{-}$is lower semi-continuous and the Lebesgue integrals in the definition of $S(u, g)$ depend continuously on $g^{-}$. Also, the finite set $P_{g}$ changes continuously with respect to the Hausdorff metric as $g$ changes continuously with respect to the partial distance on $\left(U \rightarrow^{u}\right.$ $\mathbf{I R})$. The result follows as $s(u, g)$ is the minimum of a finite number of functions that vary continuously with $g$.

In order to obtain the regularity results of this section, we need the following density lemma.

Lemma 6.2 (Density Lemma) Let $f \in \delta(g)$, with step function $g \in(U \rightarrow \mathbf{I} \mathbb{R})$ and let $\epsilon>0$ be given. Then there exists a step function $h$ and a function $k$ with $g \ll h$ and $k \in \delta(h)$ such that $\|f-k\|<\epsilon$.

Proposition 6.3 For any step function $g \in(U \rightarrow \mathbf{I} \mathbb{R})$ we have: $\delta(g) \subset C l(\breve{\delta}(g))$.
Proof Suppose $f \in \delta(g)$. We show that any basic L-open set containing $f$ will intersect $\breve{\delta}(g)$. Let $f \in \breve{\delta}\left(g_{0}\right)$ for some step function $g_{0}$ and consider any open ball $O_{\epsilon}(f)$ of radius $\epsilon>0$, around $f$ in the sup norm in $(U \rightarrow \mathbb{R})$. Then, $f \in$ $\delta\left(g_{1}\right)$ for some $g_{1}$ with $g_{0} \ll g_{1}$. Thus, $\mathcal{L} f \sqsupseteq g_{0}$ and $\mathcal{L} f \sqsupseteq$ $g_{1}$. Put $g_{2}:=g \sqcup g_{1}$. Then $f \in \delta\left(g_{2}\right)$. By Lemma 6.2, there exists a step function $h$ with $g_{2} \ll h$ and $k \in(U \rightarrow \mathbb{R})$ with $\|f-k\|<\epsilon$ and $k \in \delta(h)$. Thus, $g_{0} \ll h$ and $g \ll h$ and we have: $\breve{\delta}(g) \cap \breve{\delta}\left(g_{0}\right) \cap O_{\epsilon}(f) \neq \emptyset$ as required.

Recalling Corollary 4.2, we have now all together proved.

Corollary 6.4 For any step function $g \in(U \rightarrow \mathbf{I} \mathbb{R})$ we have: $\delta(g)=\operatorname{Cl}(\breve{\delta}(g))$.

Proposition 6.5 For any step function $g \in(U \rightarrow \mathbb{I} \mathbb{R})$ we have: $\delta^{\circ}(g)=\breve{\delta}(g)$.

Proof Since $\delta(g)=\mathrm{Cl}(\breve{\delta}(g))$, we already know that $\delta^{\circ}(g) \supset \breve{\delta}(g)$. To show the converse, let $f \in \delta^{\circ}(g)$, i.e., there exists a step function $h$ such that $f \in \breve{\delta}(h) \subset \delta(g)$. The latter relation implies, by Corollary 4.6(i), that for any $k$ with $h \ll k$ we have $\delta(k) \subset \delta(g)$. It follows that $h \sqsupseteq g$. On the other hand $f \in \breve{\delta}(h)$ implies there exists a step function $k$ with $h \ll k$ and $f \in \delta(k)$. Thus, $f \in \delta(k) \subset \breve{\delta}(h) \subset \breve{\delta}(g)$, where the latter relation follows from Corollary 4.6(ii).

Corollary 6.6 The basic open and closed subsets $\breve{\delta}(g)$ and $\delta(g)$ are regular open and closed sets respectively.

To extend the results of this section to higher dimensions, one has to generalise the Density lemma for $n>1$.

\section{Fundamental Theorem of Calculus}

Recall the Fundamental Theorem of Calculus of the first order in Equation 4. In this section we develop the FTC of second order in finite dimensional Euclidean spaces by constructing continuous second order typed integration and differential operators that are inverses of each other.

Throughout this section, we consider $\left(U \rightarrow \mathbf{C}\left(\mathbb{R}^{n}\right)\right)$ with its Scott topology. Since we will be dealing with the primitive maps of functions in $\left(U \rightarrow \mathbf{C}\left(\mathbb{R}^{n}\right)\right)$, we will identify maps that are almost everywhere equal in this function space. We say $f, g \in\left(U \rightarrow \mathbf{C}\left(\mathbb{R}^{n}\right)\right)$ are equivalent and write $f \equiv g$ if $f=g$ a.e., i.e. if $f(x)=g(x)$ for almost all $x \in U$ with respect to the $n$-dimensional Lebesgue measure on $U$. We denote the equivalence class of $f$ by $E(f)$. The set of equivalence classes is partially ordered by defining $E(f) \sqsubseteq E(g)$ if $f \sqsubseteq g$ a.e. It is easy to check that this partial order of equivalence classes, which we denote by $\left(U \rightarrow_{c} \mathbf{C}\left(\mathbb{R}^{n}\right)\right)$, is directed complete and the map $E:\left(U \rightarrow \mathbf{C}\left(\mathbb{R}^{n}\right)\right) \rightarrow\left(U \rightarrow_{c} \mathbf{C}\left(\mathbb{R}^{n}\right)\right)$, which takes a map to its equivalence class is Scott continuous. 
Proposition 7.1 Any equivalence class of maps has a lub in $\left(U \rightarrow \mathbf{C}\left(\mathbb{R}^{n}\right)\right)$ which is in the same class.

Proof Consider an equivalence class $E(f)$. We claim that it has a lub. Since $\left(U \rightarrow \mathbf{C}\left(\mathbb{R}^{n}\right)\right)$ is a continuous Scott domain, it is sufficient to show that $E(f)$ is a bounded set; this follows if we prove that any finite set of maps in $E(f)$ has a lub. In fact, we show that any two members of $E(f)$ have a lub in $E(f)$, from which the claim follows. Let $g \equiv f$ and for the sake of a contradiction, suppose $f(x) \cap g(x)=\emptyset$ for some $x \in U$. Then, by the Scott continuity of $f$ and $g$, there would exist an open neighbourhood of $x$ that is mapped by $f$ and $g$ to two disjoint open subsets containing $f(x)$ and $g(x)$ respectively. But this contradicts the assumption that $f=g$ a.e. It remains to show that $\bigsqcup E(f) \in E(f)$. Since $E(f)$ is a directed set, by the Scott continuity of the map $r$ of Equation 5, we have $r(\bigsqcup E(f))=r(f)$. From proposition 2.5(iii), we obtain: $\bigsqcup E(f)=f$ a.e.

Let $F:\left(U \rightarrow_{c} \mathbf{C}\left(\mathbb{R}^{n}\right)\right) \rightarrow\left(U \rightarrow \mathbf{C}\left(\mathbb{R}^{n}\right)\right)$ be the map which takes any equivalence class $E(f)$ to its lub, i.e. $F(E(f))=\bigsqcup E(f)$. We have the following.

Proposition 7.2 The pair $(F, E)$ is a continuous sectionretraction pair, with $F \circ E \sqsupseteq I d$, i.e., it is a continuous insertion-closure operation.

Corollary 7.3 The dcpo $\left(U \rightarrow_{c} \mathbf{C}\left(\mathbb{R}^{n}\right)\right)$ is a continuous $S$ cott domain and $E$ preserves the way-below relation.

Proof By [1, Theorem 3.14 and Proposition 3.1.14].

Thus, $\left(U \rightarrow_{c} \mathbf{C}\left(\mathbb{R}^{n}\right)\right)$ is, by identifying it with its image under the map $F$, in effect a continuous Scott sub-domain of $\left(U \rightarrow \mathbf{C}\left(\mathbb{R}^{n}\right)\right)$. We know that $f=g$ a.e. implies that $\int f=\int g$, therefore elements of the same class have the same primitive maps. We conclude that taking quotients under the equivalence relation of equality almost everywhere preserves the domain-theoretic structure. Therefore, we adapt the same convention as in classical measure theory where maps that are almost everywhere equal are identified. This means that from now on we implicitly consider $g \in\left(U \rightarrow \mathbf{C}\left(\mathbb{R}^{n}\right)\right)$ as an equivalence class of maps and all relations between maps are assumed to be between their equivalence classes. Therefore $f=g$ means that $f$ and $g$ are in the same equivalence class. i.e., $f=g$ a.e.

To deal with the primitive maps of $\left(U \rightarrow \mathbf{C}\left(\mathbb{R}^{n}\right)\right)$, we still need to restrict to a smaller subdomain, namely that of the integrable maps, i.e. $f \in\left(U \rightarrow \mathbf{C}\left(\mathbb{R}^{n}\right)\right)$ with $\int f \neq \emptyset$. The integrable maps of $\left(U \rightarrow \mathbf{C}\left(\mathbb{R}^{n}\right)\right)$ form a Scott closed subset, and thus a continuous Scott subdomain of $\left(U \rightarrow \mathbf{C}\left(\mathbb{R}^{n}\right)\right)$ [17]. By taking retraction under the restrictions of $E$ and $F$, we obtain the Scott continuous domain of equivalence classes of integrable maps which we denote by $\left(U \rightarrow_{i} \mathbf{C}\left(\mathbb{R}^{n}\right)\right)$.
Let $T^{*}(U)$ be the dcpo of non-empty ties. Define

$$
\hat{\mathcal{L}}: T^{*}(U) \rightarrow\left(U \rightarrow{ }_{i} \mathbf{C}\left(\mathbb{R}^{n}\right)\right)
$$

by $\hat{\mathcal{L}}(\Delta)=\prod\{\mathcal{L} h: h \in \Delta\}$.

Proposition 7.4 The integral map $\int:\left(U \rightarrow_{i} \mathbf{C}\left(\mathbb{R}^{n}\right)\right) \rightarrow$ $T^{*}(U)$ and $\hat{\mathcal{L}}$ are inverses of each other.

Let $C_{i}^{0}(U)$ denote the set of integrable $C^{0}$ real-valued vector fields of type $U \rightarrow \mathbb{R}^{n}$ on the open subset $U \subset \mathbb{R}^{n}$ equipped with the subspace $C^{0}$ topology. Let $\left\{C^{1}(U)\right\}$ be the equivalence classes of real-valued $C^{1}$ maps on $U$ under the equivalence relation $f \backsim g$ if $f-g$ is a constant real number. Then, $\left\{C^{1}(U)\right\}$ inherits the $C^{1}$ norm topology. Let $\mathcal{I}_{1}$ and $\mathcal{I}_{0}$ be respectively the insertion of $\left\{C^{1}(U)\right\}$ and $C_{i}^{0}(U)$ into the maximal elements of the continuous Scott domains $T^{*}(U)$ and $\left(U \rightarrow_{i} \mathbf{C}\left(\mathbb{R}^{n}\right)\right)$. These insertions are topological embeddings with respect to the Scott topology on the two continuous Scott domains.

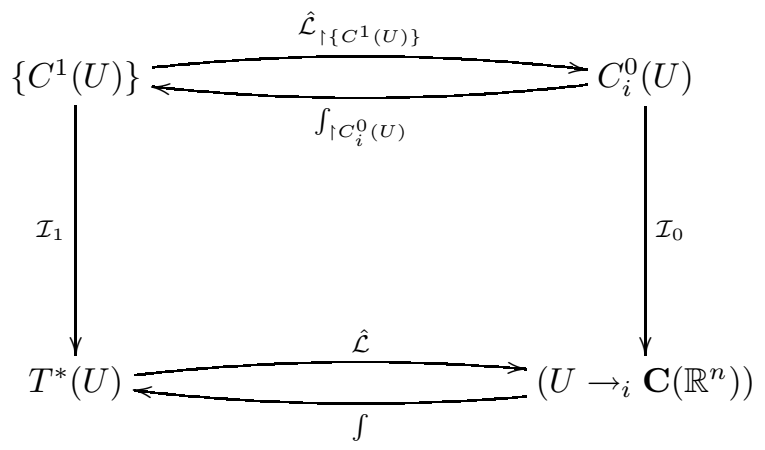

Corollary 7.5 Second order typed FTC The Scott continuous maps $\hat{\mathcal{L}}$ and $\int$ furnish an isomorphism between the Scott continuous domains for ties and L-derivatives, extending the Fundamental Theorem of Calculus of second order via the topological embeddings $\mathcal{I}_{1}$ and $\mathcal{I}_{0}$ to Lipschitz maps.

\section{Further work and open problems}

It can be shown that in finite dimensions the L-derivative operator $\mathcal{L}:(U \rightarrow \mathbb{R}) \rightarrow\left(U \rightarrow \mathbf{C}\left(\mathbb{R}^{n}\right)\right)$ is onto. It then follows that $\left((U \rightarrow \mathbb{R}) \rightarrow\left(U \rightarrow \mathbf{C}\left(\mathbb{R}^{n}\right)\right)\right)$, where $(U \rightarrow \mathbb{R})$ is equipped with the L-topology, is a continuous Scott domain. This function space can then be given an effective structure and $\mathcal{L}$ becomes a computable operator.

We list here three open questions: (i) Can the specific results for dimension one in Section 6, i.e. regularity of the basic open subsets, be extended to any finite dimension? (ii) How can the Scott topology on $\left(U \rightarrow \mathbf{C}\left(X^{*}\right)\right)$ and thus the L-topology be characterised for Lipschitz maps on an infinite dimensional Banach space $X$ ? Can any of the results in finite dimension be extended to infinite dimensions? (iii) Can one construct a simple complete metric for 
the L-topology by using the Hausdorff metric to compare L-derivatives?

Finally, a comparison of the notions of L-derivative and strong derivative as in [4] is in order.

\section{References}

[1] S. Abramsky and A. Jung. Domain theory. In S. Abramsky, D. M. Gabbay, and T. S. E. Maibaum, editors, Handbook of Logic in Computer Science, volume 3. Clarendon Press, 1994.

[2] Y. Benyamini and J. Lindenstrauss. Geometric Nonlinear Functional Analysis. American Mathematical Society, 2000.

[3] D Burago. A Course in Metric Geometry. American Mathematical Society, 2001.

[4] R. Cazacu and J. D. Lawson. Quasicontinuous functions, domains, extended calculus, and viscosity solutions. Applied General Topology, 8:1-33, 2007.

[5] F. H. Clarke. Optimization and Nonsmooth Analysis. Wiley, 1983.

[6] F. H. Clarke, Yu. S. Ledyaev, R. J. Stern, and P. R. Wolenski. Nonsmooth Analysis and Control Theory. Springer, 1998.

[7] E. A. Coddington and N. Levinson. Theory of Ordinary Differential Equations. McGraw-Hill, 1955.

[8] T. A. Davis and K. Sigmon. MATLAB Primer. CRC Press, seventh edition, 2005.

[9] R. Devaney. An Introduction to Chaotic Dynamical Systems. Addison Wesley, second edition, 1989.

[10] A. Edalat. Domain theory and integration. Theoretical Computer Science, 151:163-193, 1995.

[11] A. Edalat. Dynamical systems, measures and fractals via domain theory. Information and Computation, 120(1):3248, 1995.

[12] A. Edalat. A continuous derivative for real-valued functions. In S. B. Cooper, B. Löwe, and A. Sorbi, editors, New Computational Paradigms, Changing Conceptions of What is Computable, pages 493-519. Springer, 2008.

[13] A. Edalat. Weak topology and a differentiable operator for Lipschitz maps. Available from the following URL: www. doc.ic.ac.uk/ ae/papers/lipschitz.ps, 2008.

[14] A. Edalat and R. Heckmann. A computational model for metric spaces. Theoretical Computer Science, 193(1-2):5373, 1998.

[15] A. Edalat, M. Krznarić, and A. Lieutier. Domain-theoretic solution of differential equations (scalar fields). In Proceedings of MFPS XIX, volume 83 of Electronic Notes in Theoretical Computer Science, 2003. Full paper in www. doc.ic.ac.uk/ ae/papers/scalar.ps.

[16] A. Edalat and A. Lieutier. Domain theory and differential calculus (Functions of one variable). Mathematical Structures in Computer Science, 14(6):771-802, December 2004.
[17] A. Edalat, A. Lieutier, and D. Pattinson. A computational model for multi-variable differential calculus. In V. Sassone, editor, Proc. FoSSaCS 2005, volume 3441 of Lecture Notes in Computer Science, pages 505-519, 2005. Full paper in "www. doc.ic.ac.uk/ ae/papers/multi.ps".

[18] A. Edalat and D. Pattinson. Domain theoretic solutions of initial value problems for unbounded vector fields. In M. Escardó, editor, Proc. MFPS XXI, volume 155, pages 565-581, 2005.

[19] A. Edalat and D. Pattinson. Inverse and implicit functions in domain theory. In P. Panangaden, editor, Proc. 20th IEEE Symposium on Logic in Computer Science (LICS 2005), pages 417-426, 2005.

[20] A. Edalat and D. Pattinson. A domain theoretic account of Euler's method for solving initial value problems. In J. Dongarra, K. Madsen, and J. Wasniewski, editors, Proc. PARA 2004, volume 3732 of Lecture Notes in Comp. Sci., pages 112-121, 2006.

[21] A. Edalat and D. Pattinson. Denotational semantics of hybrid automata. Journal of Logic and Algebraic Programming, 73:3-21, 2007.

[22] A. Edalat and D. Pattinson. A domain-theoretic account of Picard's theorem. LMS Journal of Computation and Mathematics, 10:83-118, 2007.

[23] T. Erker, M. Escardó, and K. Keimel. The way-below relation of function spaces over semantic domains. Topology and Its Applications, 89(1-2):61-74, November 1998.

[24] H. Federer. Geometric Measure Theory. Springer, 1969.

[25] G. Gierz, K. H. Hofmann, K. Keimel, J. D. Lawson, M. Mislove, and D. S. Scott. Continuous Lattices and Domains. Cambridge University Press, UK, 2003.

[26] R. B. Kirk. Sets which split families of measurable sets. American Mathematical Monthly, 79:884-886, 1972.

[27] J. D. Lawson. Spaces of maximal points. Mathematical Structures in Computer Science, 7(5):543-555, 1997.

[28] J. D. Lawson. Computation on metric spaces via domain theory. Topology and its applications, 85:247-263, May 1998.

[29] G. Lebourg. Generic differentiability of lipschitzian functions. Transaction of AMS, 256:125-144, 1979.

[30] M. B. Pour-El and J. I. Richards. Computability in Analysis and Physics. Springer-Verlag, 1988.

[31] W. Rudin. Principles of Mathematical Analysis. McGrawHill, 1964.

[32] D. R. Sherbert. Banach algebras of lipschitz functions. Pacific Journal of Mathematics, 13(4):1387-1399, 1963.

[33] V. Stoltenberg-Hansen, I. Lindström, and E. R. Griffor. Mathematical Theory of Domains, volume 22 of Cambridge Tracts in Theoretical Computer Science. Cambridge University Press, 1994.

[34] K. Weihrauch. Computable Analysis (An Introduction). Springer, 2000. 